\title{
Chronic atrophic gastritis: an update on diagnosis
}

\author{
Adriana Botezatu, Nicolae Bodrug
}

"Nicolae Testemitanu" State University of Medicine and Pharmacy, Chisinau, Republic of Moldova

DOI: $10.15386 / \mathrm{mpr}-1887$

Manuscript received: 01.09.2020

Received in revised form: 07.10 .2020

Accepted: 23.10.2020

Address for correspondence:

adriana.botezatu@usmf.md

This work is licensed under a Creative Commons Attribution-NonCommercialNoDerivatives 4.0 International License

\begin{abstract}
Background and aim. Atrophic gastritis is a precancerous gastric lesion, therefore its early detection is a priority in preventing gastric cancer. The aim of the present paper is to develop a narrative synthesis of the present knowledge on diagnostic methods of chronic atrophic gastritis.

Methods. A literature search was carried out on main databases: PubMed, Hinari, SpringerLink and Scopus (Elsevier) for the period 2000-2020. The searched keywords were: chronic atrophic gastritis, intestinal metaplasia and dysplasia + diagnosis. Inclusion criteria were focused on the articles about the invasive and non-invasive diagnosis of chronic atrophic gastritis and of precancerous gastric lesions, intestinal metaplasia and dysplasia; exclusion criteria were articles published before 2000 and those that did not include the proposed theme.

Results. The search returned 575 papers addressing the topic of precancerous lesions. From these, 60 articles were qualified representative for the materials published on the topic of this synthesis article, being those that met the inclusion criteria. The data emphasize the need to use upper digestive endoscopy with biopsies for the diagnosis of chronic atrophic gastritis. However serological diagnosis is available as alternative mainly recommended in follow up.

Conclusions. There are two main methodological approaches for the evaluation of chronic atrophic gastritis as a precancerous gastric lesions: invasive examination, which requires histological analysis of biopsy samples taken during upper digestive endoscopy, being the "gold standard" for diagnosis, and non-invasive serological examination using markers of gastric function.
\end{abstract}

Keywords: chronic atrophic gastritis, gastric cancer, Helicobacter pylori

\section{Introduction}

Gastric cancer (GC) is one of the most common gastrointestinal neoplasias worldwide with high morbidity and mortality [1-5]. The histopathological cascade for the development of intestinal GC, also called Correa cascade of gastric carcinogenesis, is a stepped process: from normal gastric epithelium to chronic non-atrophic gastritis, chronic atrophic gastritis (CAG), intestinal metaplasia (IM), dysplasia and CG [1-3,6,7]. Thus, patients with premalignant gastric lesions (CAG, IM and dysplasia) have a considerable risk of developing GC, early detection of these lesions being important for early treatment and screening for GC (Figure 1) $[2,8,9]$.

$\mathrm{CAG}$, an important stage in this sequence, is characterized by loss of gastric glands and can be evaluated endoscopically, histologically and serologically $[4,10]$. Upper digestive endoscopy (UDE) with biopsy is the "gold standard" for the diagnosis of CAG, but this procedure is invasive, the accuracy of pathological biopsy depends largely on the endoscopist's skill in obtaining multiple biopsy fragments, which,though more accurate, is not always available $[4,6,9-15]$. 


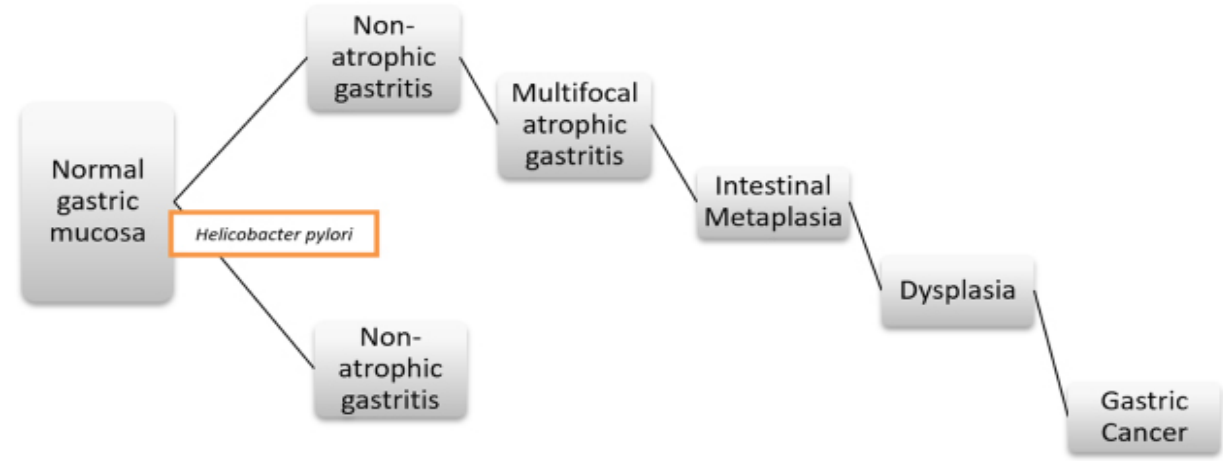

Figure 1. Schematic representation of the sequential steps of precancerous gastric lesions cascade.

With the exception of endoscopic visual inspection or histological diagnosis using updated Sydney systems, OLGA/OLGIM (Operative Link on Gastritis Assessment/ Operative Link on Gastric Intestinal Metaplasia Assessment) for CAG or IM, serum pepsinogens (PGs), also called "serological biopsy", are non-invasive diagnostic biomarkers for CAG $[6,8,9,16]$.

Simultaneously with the increase of the severity of the atrophy of the mucous glands in the antrum and/or the gastric body, the PG-I and PG-II levels change accordingly and result in a decrease of the PG-I / PG-II (PGR) ratio. Although various values have been suggested, $\mathrm{PG}-\mathrm{I} \leq 70$ $\mathrm{ng} / \mathrm{ml}$ and $\mathrm{PGR} \leq 3$ have been widely accepted for CAG or GC prediction $[8,9,11,14,16]$.

\section{The diagnosis of chronic atrophic gastritis}

There are two main methodological approaches to CAG assessment:

1. Invasive examination - during UDE biopsy samples will be taken for histological analysis, being the "gold standard" for diagnosis $[4,6,9,10,14,15]$.

2. Non-invasive serological examination using markers of gastric function: PG-I, PG-II or PG-I / PG-II ratio (PGR) with determination of gastrin 17 (G-17) and anti-Helicobacter pylori antibodies (HP-IgG).

Both the non-invasive method (serological examination) and the invasive method (endoscopic / histological examination) provide indications for the quantification of atrophic gastric changes for the patients with a potential risk of developing GC [15].

\section{Upper digestive endoscopy and histological examination}

The diagnosis of CAG can only be established by histological examination - one of the key and reliable methods for detecting atrophic, inflammatory and destructive changes of the gastric mucosa. UDE is essential for the examination of the gastric mucosa and for obtaining multiple biopsy fragments. Although endoscopic diagnosis of CAG can contribute to risk stratification and personalized screening for GC, the sensitivity of conventional endoscopy in diagnosing CAG is only $42 \%$, the accuracy of the pathological biopsy depends largely on the endoscopist's skill in obtaining multiple biopsy fragments, which, though more accurate, is not always available $[12,13]$.

There are several reports on the relationship between endoscopic and histological changes in CAG. According to the results of a study, the sensitivity and specificity of conventional white light endoscopy for the diagnosis of gastric mucosal atrophy (GMA), assessed on the basis of histological diagnosis, were $61.5 \%$ and $57.7 \%$ in the antrum, $46.8 \%$ and $76.4 \%$ in the gastric body [17]. A more recent study reported that endoscopically visible lesions (by conventional white light endoscopy) were poor predictors for HP infection or gastric precancerous disease: the positive and negative predictive values for HP were $51.5 \%$ and $49.8 \%$, respectively, for metaplastic CAG $33.3 \%$ and $72.3 \%$, respectively [18].

Conventional white light endoscopy cannot accurately diagnose and differentiate precancerous gastric lesions. Classical studies of conventional white light endoscopy have found that the correlation between histological and endoscopic changes in the diagnosis and surveillance of precancerous gastric lesions remains far from satisfactory. The detection of premalignant gastric lesions in daily practice is further based on the histological evaluation of targeted (standardized) biopsies obtained during conventional endoscopy. Untargeted biopsies should be obtained mainly from the antrum, angulus and lesser curvature of the gastric body $[1,17,19]$.

However, recent studies of conventional highdefinition white light endoscopy have shown promising results. For precancerous lesions, the method had an overall accuracy of $88 \%$ for the diagnosis of IM with a sensitivity of $74.6 \%$ and specificity of $94 \%[1,20]$. In two prospective multicenter studies, the overall accuracy of this method was $88 \%$, with a specificity of $98 \%$ for IM, but with a low 
sensitivity - 53-59\%. For the diagnosis of premalignant lesions, these two studies showed low sensitivity - $74 \%$ and $29 \%$, respectively, although the specificity was higher - $95 \%$ $[1,21,22]$. Conventional high-definition white light endoscopy with magnification may improve these results, but such data are too scarce to draw definitive conclusions $[1,23]$.

Morphological changes of the gastric mucosa, infected with HP, can be reliably identified using conventional narrowband magnification endoscopy, which can also predict the histopathological severity of CAG [24]. The diagnostic accuracy for the determination of normal gastric mucosa was 0.87 , for gastritis associated with HP infection - 0.65 and for GMA - 0.88. The method was superior to serological tests for the detection of GMA: 0.88 and $0.74(\mathrm{PGR}<3 ; \mathrm{p}<0.0001), 0.88$ and 0.75 (PG-I $<30 \mu \mathrm{g} / \mathrm{l} ; \mathrm{p}<0.0001)$ and white light endoscopy with magnification ( 0.88 and $0.81 ; \mathrm{p}<0.0001)$ [25].

Second-generation narrowband endoscopy provides more accurate results and significantly increases the diagnostic efficiency of advanced gastric premalignant lesions (IM, dysplasia) and early GC compared to conventional high-definition white light endoscopy $[1,22,26-28]$. If the specificity for IM and CG were similar, then the sensitivity for IM $(92 \%$ vs. $59 \%)$ and for CG $(100 \%$ vs. $29 \%)$ had a much greater difference in second-generation narrowband endoscopy compared to white light endoscopy $[1,22]$. Narrowband magnification endoscopy allows the evaluation of the gastric mucosa pattern and the microvascular pattern of gastric lesions, is useful in diagnosing and differentiating focal lesions of the gastric mucosa and in facilitating the targeting of biopsies sampling sites [1,26,27]. Targeted biopsies or mapping biopsies on narrowband endoscopy had a 2-fold higher yield in detecting IM in patients at high risk for GC compared to biopsies guided by conventional highdefinition white light endoscopy $[1,28]$.

Therefore, conventional white light and high definition endoscopy offers a wide spectrum of sensitivity $(42.4-80 \%)$ and specificity $(67-100 \%)$ in IM detection. Narrowband endoscopy technology has consistently demonstrated an improved sensitivity of approximately 70 $89 \%$. However, the specificity varied considerably - between $58 \%$ and $93 \%$. Magnified endoscopy with or without narrowband/chromoendoscopy has a significantly higher sensitivity (80-94\%) and specificity $(93-100 \%)[1,20]$.

However, UDE is a subjective invasive diagnostic tool that requires expensive equipment, considerable professional experience and is not suitable for populationbased screening for GC [29].

\section{Serological examination}

Since 2003, the GastroPanel ${ }^{\circledR}$ serological panel has been discussed, it contains 4 biomarkers of blood serum (PG-I, PG-II, G-17 and HP-IgG) and is designed to provide information about the structure and function of the gastric mucosa. In addition to indicating the functional or structural nature of the lesion, GastroPanel ${ }^{\circledR}$ is able to determine its location - the antrum or gastric body. The major areas of use of the serological panel are: 1) first-line diagnosis in patients with dyspeptic symptoms and 2) screening of asymptomatic individuals for the risk of GC (HP and CAG). This is a promising non-invasive and useful method for diagnosing CAG, with the ability to diagnose and locate different degrees of atrophy. Several studies have confirmed the usefulness of the GastroPanel $\mathbb{\circledR}$ test as a "serological biopsy" in the non-invasive assessment of CAG in patients with dyspepsia, and therapeutic procedures or decisions are made based on these results [29-34].

The results of the GastroPanel $\AA$ test are interpreted by a special software (GastroSoft $\AA)$, optimized for the use of gastritis classification according to the updated Sydney system, with the interpretation of the results in five diagnostic categories, reflecting gastric morphology: 1) normal gastric mucosa, 2) chronic non-atrophic gastritis (HP), 3) antral CAG, 4) CAG in the body and 5) CAG in the antrum and body (atrophic pangastritis) [7,29,3234]. For HP infection there are three possible outcomes: a) active HP infection (HP-IgG $>30$ immune fermentative units with all other markers in a normal range), although an active and continuous HP infection can cause a severe inflammatory reaction with serum levels of any or all three biomarkers, b) successful eradication of HP (normalization of the values of the three markers with a delay of a few weeks, and HP-IgG levels may remain high for a longer period of time) and c) failed eradication of HP (slightly elevated levels of HP-IgG, PG-II and/or G-17 - a sign of a continuous inflammatory process) [7,29,32-34].

Determination of PG-I and PG-II levels in blood serum and PGR value is used as a screening method for the detection of CAG and GC. The sensitivity and specificity of this method are relatively low $(84.6 \%$ and $73.5 \%$, respectively) $[4,6,8,9,14,15]$. Serological tests for these biomarkers are non-invasive, low cost and convenient compared to non-serological tests such as UDE and histological investigations [14-16,21,30,31].

Serum PGs (PG-I and PG-II) are proenzymes of pepsin, an endoproteinase present in gastric juice. PG-I is secreted exclusively by the main cells and mucous cells of the fundus glands and gastric body, and PG-II is secreted by all gastric glands and proximal glands of the duodenal mucosa (Brunner's glands). Only 1\% of PG secreted into the gastric lumen is detected in serum. G-17 is secreted by $\mathrm{G}$ cells in the pyloric part of the gastric antrum and at lower rate by $\mathrm{G}$ cells in the duodenum. The main function of G-17 is to stimulate the secretion of gastric acid by the parietal cells of the gastric body, as well as to increase the motility of the gastric antrum. Thus, serum PG levels, primarily the combination of PG-I and PGR, with or without G-17 and HP-IgG determination, can be used to reflect morphological aspects, the functional (secretory) state of the gastric mucosa and to predict the development of GC $[4,6-11,14$ $16,29,32-35]$. 
The secretory capacity of the gastric mucosa is usually intact if there is no infection or an acute HP infection [35]. PG-I and PGR levels decrease in gastric body atrophy and multifocal CAG, but remain within a normal range in all other conditions. This reduction is progressive and closely correlates with CAG severity. Elevated PG-II levels reflect mucosal inflammation, the highest values being detected in non-atrophic gastritis associated with HP infection $[29,32,34,36]$. The G-17 value is higher in body atrophy, caused by hypo- or achlorhydria. The same situation applies when acid production is inhibited by prolonged use of proton pump inhibitors. When the antral mucosa is atrophied and G cells disappear, G-17 secretion remains very low, even after protein stimulation, a method that increases the performance of this test. In body and antral CAG, PG-I, PG-II and G-17 are substantially reduced $[29,32,34,37]$. However, due to the low sensitivity of the G-17 test, scientists do not recommend the separate use of serum G-17 for screening of antral CAG, but only in combination with serum PG and HP-IgG [37].

Therefore, the loss of glandular cells in the gastric mucosa in CAG induces significant functional changes. Atrophy in the gastric antrum reduces G-17 secretion, and atrophy in the gastric body decreases PG-I and PGR levels. PG-II, being synthesized in all gastric glands, decreases in patients with multifocal CAG $[5,34,38]$.

Although various values have been suggested, yet $\mathrm{PG}-\mathrm{I} \leq 70 \mathrm{ng} / \mathrm{ml}$ and $\mathrm{PGR} \leq 3$ have been widely accepted for CAG or GC prediction $[8,9,14,16,35]$. A systematic review and recent meta-analysis revealed sensitivity and specificity of threshold values (PG- $\leq 70 \mathrm{ng} / \mathrm{ml}$ and $\mathrm{PGR} \leq 3)$ for the diagnosis of CAG $(0.59$ and 0.89 , respectively) and for the diagnosis of $\mathrm{GC}(0.59$ and 0.73 , respectively). Due to their high specificity, noninvasiveness and easy interpretation, serum PGs have the potential for screening CAG or GC [35].

A systematic review and meta-analysis, which included 31 studies with 1,520 patients with GC and 2,265 patients with $\mathrm{CAG}$, assessed the significance of serum PG in GC and CAG screening. The summary sensitivity and summary specificity for GC screening were 0.69 and 0.73 , and for CAG screening - 0.69 and 0.88 , respectively. Subgroup analysis found that the use of the combination of PG-I level and PGR value for GC screening increased sensitivity $(0.70)$ and specificity $(0.79)$ compared to the separate use of PG-I concentration - 0.55 and 0.79 , respectively. For CAG screening, the use of the combination of PG-I level and PGR value increased sensitivity (0.79) and specificity (0.89) compared to the separate use of PGR value (0.69 and 0.84 , respectively), and separate use of PG-I concentration (0.46 and 0.93 , respectively). Thus, serum PGs have great potential as a non-invasive, population-based screening tool in GC and CAG [3,5,39].

According to the results of recent literature reviews, HP-IgG provides significant additional diagnostic information to these 3 biomarkers. HP-IgG levels cause 2 potentially different conditions: active HP infection or previous exposure to HP. Abnormal HP-IgG alone indicates chronic non-atrophic gastritis associated with HP infection. In case of association with abnormal values of the other 3 markers, high HP-IgG levels confirm the diagnosis of CAG associated with HP infection [29,32,33]. In addition, the accuracy of combining the serum PG assay with HP-IgG and/or G-17 for CAG and GC prediction was confirmed $[8,9,14,16,35]$.

Therefore, multiple systematic reviews and metaanalyses, cohort and case-control studies support the use of PGR separately or in combination with serum PG-I for the serological definition of CAG and may be useful as a screening test for $\mathrm{GC}$ in epidemiological studies population-based [40-46]. The definition of CAG based exclusively on PGR may be even superior to the definition of CAG based only on PG-I or on the combination of PGR and PG-I $[43,44,47,48]$. The separate use of serum levels of PG-I or PG-II is not sensitive enough for the diagnosis of CAG $[31,42,43,45,48]$.

In the last 15 years, the GastroPanel ${ }^{\circledR}$ test has been validated in a large number of clinical trials and screening studies, based on confirmations of biopsies taken at endoscopy [7,29,32-34,46,47,49-51]. The excellent clinical performance of this test was also validated in two recent meta-analyses. According to the results of a meta-analysis, published in 2016, which included 27 studies published up to May 2016 with 8,654 patients from different geographical areas, GastroPanel ${ }^{\circledR}$ demonstrated a better diagnosis of body CAG than antral CAG: sensitivity $70.2 \%$ and $51.6 \%$, specificity $93.9 \%$ and $84.1 \%$, respectively. Normal values of PG-I, PG-II and PGR exclude CAG with a negative predictive value of over $95 \%$ [7,29,32,34,47,51]. Another meta-analysis, published in 2017, which brought together 20 studies with a number of 4,241 patients, evaluated the performance of the panel test for the diagnosis of CAG of any location and severity. The summary sensitivity of the test was $74.7 \%$, the summary specificity $-95.6 \%$, the negative predictive value $-91 \%$ and the positive predictive value $-86 \%$. Both the prevalence of CAG $(89.5 \%$ and $83.7 \%$, respectively) and the prevalence of HP infection ( $81.4 \%$ and $84.9 \%$, respectively) based on histological examination were similar to those based on GastroPanel ${ }^{\circledR}$ $[33,46]$.

The diagnosis of HP infection is necessary for any gastritis and is the etiological factor of CAG. There are 2 groups of methods - direct and indirect. The morphological study of biopsy specimens is recognized as a "gold standard" of reliability. Determination of HP by stool antigen test is a cheap and simple method that allows the identification of indirect signs of secretory insufficiency of the stomach [38]. But the most widely used tests (urea breath test and stool antigen test) have several limitations. These limitations are completely avoided by the GastroPanel ${ }^{\circledR}$ 
test, in which the HP-IgG determination is supplemented by 3 other biomarkers (PG-I, PG-II, G-17), which are sensitive indicators of inflammation of the gastric mucosa. This panel has no known shortcomings (false-negative and false-positive results) of conventional HP tests and has added value by detecting (with great accuracy) another major risk factor of the GC - CAG - with all its potential clinical sequelae $[5,29]$.

Therefore, the use of serum biomarkers in the diagnosis of premalignant gastric lesions presents an attractive alternative to endoscopy with histopathological examination of gastric biopsy specimens. The method is non-invasive, involves simple blood sampling and provides consistent results [44]. The non-invasive GastroPanel ${ }^{\circledR}$ test strongly correlates with the results of the histological examination and should be used to differentiate with high precision subjects with healthy gastric mucosa and those with lesions of the gastric mucosa. The test is a reliable tool for the diagnosis of CAG, which should be used as a first line for the diagnosis and screening of people or populations at high risk for GC [3,4,7,9,29,32,33,46,47,52,53].

However, the authors of a study, published in 2014, concluded that the serological panel is not accurate enough for the diagnosis of CAG and its systematic use in clinical practice cannot be recommended [54]. Moreover, the guidelines of the British Society of Gastroenterology also consider that although biomarkers help to detect patients with CAG, there is not enough evidence to support their use in population screening. High definition endoscopy combined with biopsy sampling and histopathological examination is the best test for accurate detection and stratification of CAG and IM risk. However, there is insufficient evidence to support endoscopic screening in a population at low risk of GC, but endoscopic surveillance every 3 years should be provided to patients with extensive CAG and IM [3].

Comparison of endoscopic/histological and serological examinations

Reports on the value of endoscopy for the diagnosis of CAG are still controversial. However, the endoscopic method, histological examination and serological diagnostic tests of CAG correlate relatively well $[55,56]$. The value of PGR separately and in combination with PG-I decreased significantly $(\mathrm{p}<0.001)$ simultaneously with the increase of the degree of GMA and the threshold value of distinction between the presence and absence of endoscopic CAG was 3.0-3.2 [55].

Serum PG levels correlate well with abnormal endoscopic findings. PGR was inversely correlated with CAG (transparency of blood vessels with a demarcation line - atrophic border) and IM (whitish spots with or without depressed hyperemic lesions) $(p<0.001)$ [57]. PGR is closely correlated with histological CAG, and PGR $<3.0$ is considered an optimal value for the diagnosis of $\mathrm{CAG}$ with high sensitivity (71\%), specificity (86\%) and PPV (85\%) [11]. A significant correlation was found between CAG, determined endoscopically and histologically, and the sensitivity and specificity of the endoscopic diagnosis were $65.9 \%$ and $58.0 \%$ for the gastric antrum, $71.3 \%$ and $53.7 \%$ for the gastric body, respectively [55].

The PGR value separately and in combination with the serum level of PG-I correlates statistically significantly inversely with the OLGA and OLGIM staging systems $[1,15,58-60]$ and these differences were more pronounced in the high-risk stages (OLGA/OLGIM III-IV) [58,59]. Low serum PG-I levels, especially when associated with HP-negative serological status, may identify patients at higher risk for $\mathrm{GC}$, requiring endoscopic evaluation and monitoring [1].

Therefore, several studies have shown that histological staging and serological stratification have similar important roles in predicting precancerous lesions, and their staging, including OLGA and OLGIM systems, combined with serum PG levels, can provide important information for GMA and IM risk assessment. Serum PG-I levels and PGR values, especially PGR, are valuable markers of changes in the gastric mucosa. Moreover, G-17 levels and serum PG levels may provide important clinical data in identifying the area of atrophy [58]. However, the sensitivity and specificity of endoscopic findings and serological results for the diagnosis of CAG are not high, and the histological diagnosis has some limitations. Thus, given the limitations of these three methods, a multifactorial evaluation is needed to improve the accuracy of CAG diagnosis [55]. Therefore, for accurate prediction of $\mathrm{GC}$ risk in clinical practice, $\mathrm{CAG}$ staging, including OLGA and OLGIM systems, must be combined with serum PG values [58].

\section{Conclusions}

There are two main methodological approaches for the evaluation of chronic atrophic gastritis: 1) invasive examination, which requires histological analysis of biopsy samples taken during upper digestive endoscopy, being the "gold standard" for diagnosis; 2) non-invasive serological examination using markers of gastric function (serum pepsinogens I, II and pepsinogen ratio I/II with determination of gastrin 17 and anti-Helicobacter pylori antibodies. Upper digestive endoscopy is essential for the examination of the gastric mucosa and for obtaining multiple biopsy fragments. Simultaneously, endoscopic diagnosis of chronic atrophic gastritis can contribute to risk stratification and personalized screening for gastric cancer, and the implementation of GastroPanel ${ }^{\circledR}$ test in clinical practice would be of significant importance in the early diagnosis of chronic atrophic gastritis among people at high risk of gastric cancer and lead to better overall patient survival rates. 


\section{References}

1. Pimentel-Nunes P, Libânio D, Marcos-Pinto R, Areia M, Leja M, Esposito G, et al. Management of epithelial precancerous conditions and lesions in the stomach (MAPS II): European Society of Gastrointestinal Endoscopy (ESGE), European Helicobacter and Microbiota Study Group (EHMSG), European Society of Pathology (ESP), and Sociedade Portuguesa de Endoscopia Digestiva (SPED) guideline update 2019. Endoscopy. 2019;51:365-388.

2. Koulis A, Buckle A, Boussioutas A. Premalignant lesions and gastric cancer: Current understanding. World J Gastrointest Oncol. 2019;11:665-678.

3. Banks M, Graham D, Jansen M, Gotoda T, Coda S, di Pietro $\mathrm{M}$, et al. British Society of Gastroenterology guidelines on the diagnosis and management of patients at risk of gastric adenocarcinoma. Gut. 2019;68:1545-1575.

4. Lahner E, Zagari RM, Zullo A, Di Sabatino A, Meggio A, Cesaro P, et al. Chronic atrophic gastritis: Natural history, diagnosis and therapeutic management. A position paper by the Italian Society of Hospital Gastroenterologists and Digestive Endoscopists [AIGO], the Italian Society of Digestive Endoscopy [SIED], the Italian Society of Gastroenterology [SIGE], and the Italian Society of Internal Medicine [SIMI]. Dig Liver Dis. 2019;51:1621-1632.

5. Chapelle N, Petryszyn P, Blin J, Leroy M, Le Berre-Scoul C, Jirka I, et al. A panel of stomach-specific biomarkers (GastroPanel ${ }^{\circledR}$ ) for the diagnosis of atrophic gastritis: A prospective, multicenter study in a low gastric cancer incidence area. Helicobacter. 2020;25:e12727.

6. Valdes-Socin H, Leclercq P, Polus M, Rohmer V, Beckers A, Louis E. Chronic autoimmune gastritis: a multidisciplinary management. Rev Med Liege. 2019;74:598-605.

7. Syrjänen K, Eskelinen M, Peetsalu A, Sillakivi T, Sipponen P, Härkönen $M$, et al. GastroPanel ${ }^{\circledR}$ Biomarker Assay: The Most Comprehensive Test for Helicobacter pylori Infection and Its Clinical Sequelae. A Critical Review. Anticancer Res. 2019;39:1091-1104.

8. Bang CS, Lee JJ, Baik GH. Diagnostic performance of serum pepsinogen assay for the prediction of atrophic gastritis and gastric neoplasms: Protocol for a systematic review and meta-analysis. Medicine (Baltimore). 2019;98:e14240.

9. Bang CS, Lee JJ, Baik GH. Prediction of Chronic Atrophic Gastritis and Gastric Neoplasms by Serum Pepsinogen Assay: A Systematic Review and Meta-Analysis of Diagnostic Test Accuracy. J Clin Med. 2019;8:657.

10. Rodriguez-Castro KI, Franceschi M, Noto A, Miraglia C, Nouvenne A, Leandro G, et al. Clinical manifestations of chronic atrophic gastritis. Acta Biomed. 2018;89(Suppl 8): 88-92.

11. Su W, Zhou B, Qin G, Chen Z, Geng X, Chen X, et al. Low PG I/II ratio as a marker of atrophic gastritis: Association with nutritional and metabolic status in healthy people. Medicine (Baltimore). 2018;97:e10820.

12. Zhang Y, Li F, Yuan F, Zhang K, Huo L, Dong Z, et al. Diagnosing chronic atrophic gastritis by gastroscopy using artificial intelligence. Dig Liver Dis. 2020;52:566-572.
13. Jin E, Chung SJ, Lim JH, Chung GE, Lee C, Yang JI, et al. Training Effect on the Inter-observer Agreement in Endoscopic Diagnosis and Grading of Atrophic Gastritis according to Level of Endoscopic Experience. J Korean Med Sci. 2018;33:e117.

14. Korstanje A, den Hartog G, Biemond I, Lamers C. Chapter III. The serological gastric biopsy: a non-endoscopical diagnostic approach in management of the dyspeptic patient. Scand J Gastroenterol. 2002;7(suppl 236):22-26 (modified version).

15. Mescoli C, Gallo Lopez A, Taxa Rojas L, Jove Oblitas W, Fassan M, Rugge M. Gastritis staging as a clinical priority. Eur J Gastroenterol Hepatol. 2018;30:125-129.

16. Cha JH, Jang JS. Clinical correlation between serum pepsinogen level and gastric atrophy in gastric neoplasm. Korean J Intern Med. 2018;35:550-558.

17. Eshmuratov A, Nah JC, Kim N, Lee HS, Lee HE, Lee BH, et al. The correlation of endoscopic and histological diagnosis of gastric atrophy. Dig Dis Sci. 2010;55:1364-1375.

18. Lahner E, Esposito G, Zullo A, Hassan C, Carabotti M, Galli $\mathrm{G}$, et al. Gastric precancerous conditions and Helicobacter pylori infection in dyspeptic patients with or without endoscopic lesions. Scand J Gastroenterol. 2016;51:12941298.

19. Huang RJ, Choi AY, Truong CD, Yeh MM, Hwang JH. Diagnosis and Management of Gastric Intestinal Metaplasia: Current Status and Future Directions. Gut Liver. 2019;13:596-603.

20. Panteris V, Nikolopoulou S, Lountou A, Triantafillidis JK. Diagnostic capabilities of high-definition white light endoscopy for the diagnosis of gastric intestinal metaplasia and correlation with histologic and clinical data. Eur J Gastroenterol Hepatol. 2014;26:594-601.

21. Pimentel-Nunes P, Libânio D, Lage J, Abrantes D, Coimbra M, Esposito G, et al. A multicenter prospective study of the real-time use of narrow-band imaging in the diagnosis of premalignant gastric conditions and lesions. Endoscopy. 2016;48:723-730.

22. Ang TL, Pittayanon R, Lau JY, Rerknimitr R, Ho SH, Singh $\mathrm{R}$, et al. A multicenter randomized comparison between highdefinition white light endoscopy and narrow band imaging for detection of gastric lesions. Eur J Gastroenterol Hepatol. 2015;27:1473-1478.

23. Anagnostopoulos GK, Yao K, Kaye P, Fogden E, Fortun P, Shonde A, et al. High-resolution magnification endoscopy can reliably identify normal gastric mucosa, Helicobacter pylori-associated gastritis, and gastric atrophy. Endoscopy. 2007;39:202-207.

24. Tongtawee T, Kaewpitoon S, Kaewpitoon N, Dechsukhum C, Loyd RA, Matrakool L. Correlation between Gastric Mucosal Morphologic Patterns and Histopathological Severity of Helicobacter pylori Associated Gastritis Using Conventional Narrow Band Imaging Gastroscopy. Biomed Res Int. 2015;2015:808505.

25. White JR, Sami SS, Reddiar D, Mannath J, Ortiz-FernándezSordo J, Beg S, et al. Narrow band imaging and serology in the assessment of premalignant gastric pathology. Scand $\mathrm{J}$ Gastroenterol. 2018;53:1611-1618. 
26. Ang TL, Fock KM, Teo EK, Tan J, Poh CH, Ong J, et al. The diagnostic utility of narrow band imaging magnifying endoscopy in clinical practice in a population with intermediate gastric cancer risk. Eur J Gastroenterol Hepatol. 2012;24:362-367.

27. Uedo $\mathrm{N}$, Ishihara $\mathrm{R}$, Iishi $\mathrm{H}$, Yamamoto $\mathrm{S}$, Yamamoto $\mathrm{S}$, Yamada $\mathrm{T}$, et al. A new method of diagnosing gastric intestinal metaplasia: narrow-band imaging with magnifying endoscopy. Endoscopy. 2006;38:819-824.

28. Buxbaum JL, Hormozdi D, Dinis-Ribeiro M, Lane C, DiasSilva D, Sahakian A, et al. Narrow-band imaging versus white light versus mapping biopsy for gastric intestinal metaplasia: a prospective blinded trial. Gastrointest Endosc. 2017;86:857-865.

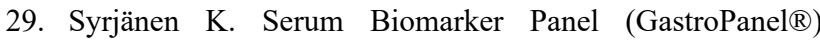
and Slow-Release L-cysteine (Acetium ${ }^{\circledR}$ Capsule): Rationale for the Primary Prevention of Gastric Cancer. EC Gastroenterology and Digestive System. 2017;3:172-192.

30. Wang X, Ling L, Li S, Qin G, Cui W, Li X, et al. The Diagnostic Value of Gastrin-17 Detection in Atrophic Gastritis: A MetaAnalysis. Medicine (Baltimore). 2016;95:e3599.

31. Dong Z, Zhang X, Chen X, Zhang J. Significance of Serological Gastric Biopsy in Different Gastric Mucosal Lesions: an Observational Study. Clin Lab. 2019;65(12). doi: 10.7754/Clin.Lab.2019.190433

32. Syrjänen K. Serological Biomarker Panel (GastroPanel®): A Test for Non-Invasive Diagnosis of Dyspeptic Symptoms and for Comprehensive Detection of Helicobacter pylori Infection. Biomark J. 2017;3:1.

33. Loor A, Dumitraşcu DL. Helicobacter pylori Infection, Gastric Cancer and Gastropanel. Rom J Intern Med. 2016;54:151-156.

34. Syrjänen K, Eronen K. Serological Testing in Management of Dyspeptic Patients and in Screening of Gastric Cancer Risks. J Gastrointest Disord Liver Func. 2016;2:84-88.

35. Kim EH, Kang H, Park CH, Choi HS, Jung DH, Chung $\mathrm{H}$, et al. The optimal serum pepsinogen cut-off value for predicting histologically confirmed atrophic gastritis. Dig Liver Dis. 2015;47:663-668.

36. Giroux V, Rustgi AK. Metaplasia: tissue injury adaptation and a precursor to the dysplasia-cancer sequence. Nat Rev Cancer. 2017;17:594-604.

37. Leja M, Kupcinskas L, Funka K, Sudraba A, Jonaitis L, Ivanauskas A, et al. Value of gastrin-17 in detecting antral atrophy. Adv Med Sci. 2011;56:145-150.

38. McNicholl AG, Forné M, Barrio J, De la Coba C, González B, Rivera R, et al. Accuracy of GastroPanel for the diagnosis of atrophic gastritis. Eur J Gastroenterol Hepatol. 2014;26:941948.

39. Huang YK, Yu JC, Kang WM, Ma ZQ, Ye X, Tian SB, et al. Significance of Serum Pepsinogens as a Biomarker for Gastric Cancer and Atrophic Gastritis Screening: A Systematic Review and Meta-Analysis. PLoS One. 2015;10:e0142080.

40. Sugano K, Tack J, Kuipers E, Graham DY, El-Omar EM, Miura S, et al. Kyoto global consensus report on Helicobacter pylori gastritis. Gut. 2015;64:1353-1367.
41. Mårdh E, Mårdh S, Mårdh B, Borch K. Diagnosis of gastritis by means of a combination of serological analyses. Clin Chim Acta. 2002;320:17-27.

42. Weck MN, Brenner H. Association of Helicobacter pylori infection with chronic atrophic gastritis: Meta-analyses according to type of disease definition. Int $\mathrm{J}$ Cancer. 2008;123:874-881.

43. Broutet N, Plebani M, Sakarovitch C, Sipponen P, Mégraud F; Eurohepygast Study Group. Pepsinogen A, pepsinogen $\mathrm{C}$, and gastrin as markers of atrophic chronic gastritis in European dyspeptics. Br J Cancer. 2003;88:1239-1247.

44. Loong TH, Soon NC, Nik Mahmud NRK, Naidu J, Rani RA, Abdul Hamid N, et al. Serum pepsinogen and gastrin-17 as potential biomarkers for pre-malignant lesions in the gastric corpus. Biomed Rep. 2017;7:460-468.

45. Mansour-Ghanaei F, Joukar F, Baghaee M, Sepehrimanesh M, Hojati A. Only serum pepsinogen I and pepsinogen I/II ratio are specific and sensitive biomarkers for screening of gastric cancer. Biomol Concepts. 2019;10:82-90.

46. Noah D, Assoumou M, Bagnaka S, Ngaba GP, Alonge IE, Palohelmo L et al. Assessing GastroPanel serum markers as a non-invasive method for the diagnosis of atrophic gastritis and Helicobacter pylori infection. Open J Gastroenterol. 2012;2:113-118.

47. Syrjänen K. A Panel of Serum Biomarkers (GastroPanel $®$ ) in Non-invasive Diagnosis of Atrophic Gastritis. Systematic Review and Meta-analysis. Anticancer Res. 2016;36:51335144.

48. Brenner H, Rothenbacher D, Weck MN. Epidemiologic findings on serologically defined chronic atrophic gastritis strongly depend on the choice of the cutoff-value. Int $\mathrm{J}$ Cancer. 2007;121:2782-2786.

49. Pasechnikov VD, Chukov SZ, Kotelevets SM, Mostovov AN, Mernova VP, Polyakova MB. Invasive and non-invasive diagnosis of Helicobacter pylori-associated atrophic gastritis: a comparative study. Scand J Gastroenterol. 2005;40:297301 .

50. Korstanje A, den Hartog G, Biemond I, Lamers CB. The serological gastric biopsy: a non-endoscopical diagnostic approach in management of the dyspeptic patient: significance for primary care based on a survey of the literature. Scand $\mathrm{J}$ Gastroenterol Suppl. 2002;236:22-26.

51. Lahner E, Carabotti M, Annibale B. Atrophic body gastritis: clinical presentation, diagnosis, and outcome. EMJ Gastroenterol. 2017;6:75-82.

52. Zagari RM, Rabitti S, Greenwood DC, Eusebi LH, Vestito A, Bazzoli F. Systematic review with meta-analysis: diagnostic performance of the combination of pepsinogen, gastrin-17 and anti-Helicobacter pylori antibodies serum assays for the diagnosis of atrophic gastritis. Aliment Pharmacol Ther. 2017;46:657-667.

53. Iijima K, Abe Y, Kikuchi R, Koike T, Ohara S, Sipponen P, et al. Serum biomarker tests are useful in delineating between patients with gastric atrophy and normal, healthy stomach. World J Gastroenterol. 2009;15:853-859.

54. Ikeda F, Shikata K, Hata J, Fukuhara M, Hirakawa Y, Ohara $\mathrm{T}$, et al. Combination of Helicobacter pylori Antibody and 
Serum Pepsinogen as a Good Predictive Tool of Gastric Cancer Incidence: 20-Year Prospective Data From the Hisayama Study. J Epidemiol. 2016;26:629-636.

55. Lee JY, Kim N, Lee HS, Oh JC, Kwon YH, Choi YJ, et al. Correlations among endoscopic, histologic and serologic diagnoses for the assessment of atrophic gastritis. J Cancer Prev. 2014;19:47-55.

56. Al-Nuaimya WM, Faisalb HM. Endoscopical and Histopathological Interpretation of Gastritis in Nineveh Province. Ann Coll Med Mosul. 2019; 41(1): 28-35.

57. Lee SP, Lee SY, Kim JH, Sung IK, Park HS, Shim CS. Link between Serum Pepsinogen Concentrations and Upper Gastrointestinal Endoscopic Findings. J Korean Med Sci.
2017;32:796-802.

58. Wang X, Lu B, Meng L, Fan Y, Zhang S, Li M. The correlation between histological gastritis staging - 'OLGA/OLGIM' and serum pepsinogen test in assessment of gastric atrophy/ intestinal metaplasia in China. Scand J Gastroenterol. 2017;52:822-827.

59. Rugge M, Genta RM, Graham DY, Di Mario F, Vaz Coelho LG, Kim N, et al. Chronicles of a cancer foretold: 35 years of gastric cancer risk assessment. Gut. 2016;65:721-725.

60. Daugule I, Sudraba A, Chiu HM, Funka K, Ivanauskas A, Janciauskas D, et al. Gastric plasma biomarkers and Operative Link for Gastritis Assessment gastritis stage. Eur J Gastroenterol Hepatol. 2011;23:302-307. 